\title{
$\angle S$ Research Square \\ Maize (Zea Mays L.) Genotypes Induce the Changes of Rhizosphere Microbial Communities
}

\author{
Yuehan Li \\ Qiqihar University \\ Zheng Qu \\ Qiqihar University \\ Weihui Xu \\ Qiqihar University \\ Wenjing Chen \\ Qiqihar University \\ Yunlong Hu \\ Qiqihar University \\ Zhigang Wang ( $\nabla$ wangzhigang@qqhru.edu.cn ) \\ Qiqihar University https://orcid.org/0000-0002-0981-6597
}

\section{Research Article}

Keywords: Maize (Zea mays L.), Rhizosphere, Genotype, Microbial community

Posted Date: December 22nd, 2021

Dol: https://doi.org/10.21203/rs.3.rs-1183026/v1

License: @ (1) This work is licensed under a Creative Commons Attribution 4.0 International License. Read Full License 


\section{Abstract}

Plant-microbe interactions affect ecosystem function, and plant species influence relevant microorganisms. However, the different genotypes of maize that shape the structure and function of the rhizosphere microbial community remain poorly investigated. During this study, the structures of the rhizosphere microbial community among three genotypes of maize were analyzed at the seedling and maturity stages using high-throughput sequencing and bioinformatics analysis. The results demonstrated that Tiannuozao $60(\mathrm{~N})$ showed higher bacterial and fungal diversity in both periods, while Junlong1217 (QZ) and Fujitai519 (ZL) had lower diversity. The bacterial community structure among the three varieties was significantly different; however, fewer differences were found in the fungal community. The bacterial community composition of $\mathrm{N}$ and QZ was similar yet different from ZL at the seedling stage. The bacterial networks of the three cultivars were more complex than the fungal networks, and the networks of the mature stages were more complex than those of the seedling stages, while the opposite was true for the fungi. FAPROTAX functional and FUNGuild functional predictions revealed that different varieties of maize were different in functional abundance at the genus level, and these differences were related to breeding characteristics. This study suggested that different maize genotypes regulated the rhizosphere bacterial and fungal communities, which would help guide practices.

\section{Introduction}

The microbial community is a vital part of the ecological environment (Delmont et al. 2011). The interaction between plants and microbial communities is an essential link in the functioning of ecosystems (Vivanco et al. 2018) and can affect agricultural ecosystems (Teste et al. 2017). Many studies have shown that the rhizosphere is a crucial zone for plant-microbe interactions (Bulgarelli et al. 2015; Müller et al. 2016; Saleem et al. 2016; Zachow et al. 2014). The rhizosphere microbes can defend plants against pathogens through competition, antagonism, or interference with host immunity to ascertain a mutualistic association with the host (Bakker et al. 2013; Lu et al. 2018; Agler et al. 2016; Mendes et al. 2013; Mendes et al. 2011; Pozo et al. 2007; Raaijmakers et al. 2016; Reinhold-Hurek et al. 2015; Ritpitakphong et al. 2016; Yu et al. 2019). They can promote plant growth by increasing nutrient availability, manufacturing plant hormones, enhancing tolerance to abiotic stresses, and adapting to environmental variations to enhance host immune functions (Etesami et al. 2014; Haney et al. 2015; Xu et al. 2015; Mansotra et al. 2015; Berg et al. 2018; Rolli et al. 2015). The structure and function of plant microbiome change with changes in stress and environmental stimuli (Gardener et al. 2001; Ferrando et al. 2015; Santos-Medellín et al. 2017; Timm et al. 2018). Plants also affect microbial communities by producing completely different detritus and excretions (Cline et al. 2016; Zhalnina et al. 2018) and have the capacity to vary soil microbiota by secreting bioactive molecules into the rhizosphere (Schlaeppi et al. 2014; Zgadzaj et al. 2016). Therefore, understanding the interaction between microbiota and plants has important agronomic significance.

Some studies have demonstrated that plant types change the microbial community under stable environmental conditions. For example, plant genotypes have specific effects on the wheat rhizosphere microbial community (Simonin et al. 2020). The investigation of the rhizosphere bacterial community of twelve rabbit-eye blueberry (RB) cultivars demonstrated that the rhizosphere of the plant cultivars affected bacterial association networks (Jiang et al. 2017). By analyzing the rhizosphere microbial community structure and activity of maize plants, the results indicated that the rhizosphere microbial community was relevant to the plant genotype (Hou et al. 2018). These studies demonstrated that plant genotype influences microbial community composition in controlled environments. 
Maize (Zea mays L.) is one of the foremost versatile emerging crops with wider adaptability under varied agroclimatic conditions (Chandel 2018). It is, therefore, essential to understand the microbial communities of maize interrhizosphere soils. This study investigated the bacterial and fungal communities of three different maize cultivars using high-throughput sequencing methods. This study aimed to explore the composition, structure, and interactions of microbial communities from different maize cultivars. We speculated that specific differences exist among different genotypes of maize in regard to rhizosphere microorganisms. We speculated that the plant host regulated the differences and the changes were related to plant characteristics. We hope that this work can provide novel insights into the understanding of the microbiological community of maize.

\section{Materials And Methods}

\section{Experimental site, crop varieties, and soil physicochemical properties}

Experimental soils were collected at a depth of $15 \mathrm{~cm}$ from three major genotypes of corn and were set up in a complete randomized design in Qiqihar, Heilongjiang Province, China (12374'90.67" E, 4740'43.17" N; altitude: 146 $\mathrm{m}$ ) on April 18, 2020, and September 13, 2020. The average annual precipitation in this area is $670.8 \mathrm{~mm}$, and the soil at the study site is sandy loam soil. A total of 54 rhizosphere soil samples ( 9 treatments $\times 3$ replicates per treatment $\times 2$ periods) were collected from the Zea mays $L$ rhizosphere. Blank soil physical and chemical properties are given in Supplementary Table S1. The volumetric method was used to measure the content of organic matter (SOC), available nitrogen (AN), and total nitrogen (TN). UV-Vis spectrophotometry was used to measure the content of total phosphorus (TP) and available phosphorus (AP). Total potassium (TK) and available potassium (AK) were determined by the inductively coupled plasma-atomic emission spectrometry (ICP-AES) method.

The different genotypes included foodstuff-type maize Fujitai519 (ZL), Junlong1217 (QZ), and Tiannuozao60 (N) in this study. The sample properties are shown in Supplementary Table S2. Rhizosphere samples were collected unbroken in a sterile bag within an ice-containing box and transported to the laboratory. The soil was removed of all impurities with tweezers, the rhizosphere soil was gently swept with a brush, and the samples were stored in a refrigerator at $4{ }^{\circ} \mathrm{C}($ Barillot et al. 2013).

\section{DNA Extraction, PCR Amplification, and Illumina MiSeq Sequencing}

In this experiment, a total of 66 samples from two periods were used for sequencing. Microbial community genomic DNA was extracted from rhizosphere soil samples using the E.Z.N.A. ® soil DNA Kit (Omega Bio-Tek, Norcross, GA, US) according to the manufacturer's instructions. Distinct regions of bacterial 16S rRNA and fungal ITS genes were amplified using primers. The hypervariable region V3-V4 of the bacterial 16S rRNA gene was amplified with primer pairs 338F (5'-ACTCCTACGGGAGGCAGCAG-3') and 806R (5'-GGACTACHVGGGTWTCTAAT-3') and the ITS gene was amplified with primer pairs ITS1F (5'-CTTGGTCATTTAGAGGAAGTAA-3') and ITS2R (5'-GCTGCGTTCTTCATCGATGC$\left.3^{\prime}\right)$ by an ABI GeneAmp® 9700 PCR thermocycler (ABI, CA, USA).

PCR amplification of the 16S rRNA gene was performed as follows (Supplementary Table S3). The PCR product was extracted from a $2 \%$ agarose gel and purified using the AxyPrep DNA Gel Extraction Kit (Axygen Biosciences, Union City, CA, USA) according to the manufacturer's instructions and quantified using a Quantus ${ }^{\mathrm{TM}}$ Fluorometer (Promega, USA). Purified amplicons were pooled in equimolar amounts and paired-end sequenced on an Illumina MiSeq PE300 platform (Illumina, San Diego, USA) according to the standard protocols by Majorbio Bio-Pharm Technology Co., Ltd. (Shanghai, China). 


\section{Molecular biological analysis and statistical analysis}

The raw 16S rRNA and ITS gene-sequencing reads were demultiplexed, quality-filtered by fastp version 0.20.0 (Chen et al. 2018), and merged by FLASH version 1.2.7 (Magoč et al. 2011). Operational taxonomic units (OTUs) with a 97\% similarity cutoff (Edgar 2013; Stackebrandt et al. 1994) were clustered using UPARSE version 7.1 (Edgar 2013), and chimeric sequences were identified and removed. The taxonomy of each OTU representative sequence was analyzed by RDP Classifier version 2.2 (Wang Q 2007) against the 16S rRNA and ITS databases using a confidence threshold of 0.7 .

Analysis of alpha diversity and beta diversity of the normalized dataset was performed using R (version 3.2.5, USA) and the Vegan package (published by Philip Dixon, 2003). Nonmetric multidimensional scaling (NMDS) was used to visualize the dissimilarities(Taguchi et al. 2005). Differentially abundant features were analyzed using Metastat analysis in R (version 3.2.5, USA). Microbial diversity and the relative abundance of different microbial taxonomic levels were assessed for differences between different groups with one-way ANOVA, a t test, or a Kruskal-Wallis $\mathrm{H}$ test using SPSS software (SPSS, Chicago, IL, USA). Graphical representations were generated with GraphPad Prism 5 (GraphPad Software, Inc., La Jolla, CA, USA).

\section{Co-occurrence network and functional prediction analysis}

Phylogenetic molecular ecological networks (pMENs) from the three cultivars were constructed to investigate microbial interactions. The process was performed using the molecular ecological network analysis pipeline (MENA, http://ieg4.rccc.ou.edu/mena/login.cgi) (Deng et al. 2012). The co-occurrence network is based on all genus levels. Correlation networks were visualized using Gephi software (Brughmans 2013). To visualize the associations in the network, we constructed a correlation matrix by calculating the possible pairwise Spearman's rank correlations (Junker et al. 2010). FAPROTAX and FunGuild software (https://github.com/UMNFuN/FUNGuild) were used to predict the metabolic function of the microbial flora(Nguyen et al. 2016; Zhou et al. 2020)based on the literature on cultured representatives. All raw sequence data are accessible in the NCBI Sequence Read Archive (SRA) database under BioProject number PRJNA775859.

\section{Results}

\section{Analysis of alpha diversity and beta diversity of the microbial community}

In total, 12 bulk soil (CK) and 54 rhizosphere soil samples were sequenced in the experiment; a profile of bacterial and fungal communities was achieved to reveal the microbial community structure of the maize rhizosphere. For the entire sampling set, a total of 3,787,073 raw bacterial sequences with an average length of 415 bp and 3,458,212 fungal sequences with an average length of $233 \mathrm{bp}$ were identified using Illumina MiSeq analysis. After a series of preprocessing steps, 1,616,406 bacterial and 2,067,318 qualified fungal reads were classified into 6632 bacterial and 1671 fungal OTUs. All sampling efforts reached the saturation plateau using rarefaction analysis. They effectively covered the total extent of almost a majority of the bacterial and fungal diversity, consistent with 97 sequence similarities in the rank abundance curve approach (Supplementary Fig. S1).

The alpha diversity of bacterial communities of different species was reflected by the Shannon index, Simpson index, and Chao 1 index and is presented in Dataset S3. The results showed that the alpha diversity (Chao) at the seedling stage of the bacterial communities in CK was significantly lower than that in $\mathrm{N}$ and $\mathrm{ZL}(P \leq 0.05)$. However, there was no significant difference in the Shannon and Simpson indices. There was a substantial difference in the 
alpha diversity at the maturity stage of bacterial communities $(P \leq 0.05)$ (Fig. 1a). For fungi, the alpha diversity (Simpson and Shannon) in the rhizosphere soil of all three genotypes of maize was completely different compared with $\mathrm{CK}$ at the seedling and maturity stages; Simpson index values of ZL and N were significantly different in both periods of the fungus $(P \leq 0.05)$ (Fig. 1d). Cultivar $\mathrm{N}$ showed higher bacterial and fungal diversity in both periods.

NMDS analysis was conducted based on Bray-Curtis distances to visualize the differences in community composition. The overall bacterial community composition was separated from before planting at the seedling and maturity stages (stress=0.078; stress $=0.079$ ). The bacterial community was separated into cultivar groups. However, it was not as distinct as CK (Fig. 1b,c). For fungi, four treatments were also clearly separated (stress=0.069; stress=0.080) (Fig. 1e,f), and bacterial communities were less distinct than fungal communities (Fig. 1b,c,e,f). The results indicated that obvious differences existed in the microbial community structure of the rhizosphere among genotypes of maize.

\section{Microbial community composition of different maize cultivars}

The soil bacterial sequences were assigned to 8 phyla (others $<0.01$ ) in $95 \%$ of corn cultivar samples and included the phyla Actinobacteria, Proteobacteria, Acidobacteriota, Chloroflexi, Firmicutes, Bacteroidota, Gemmatimonadota, and Myxococcota. Actinobacteria accounted for the most significant proportion, 35.58\%-43.86\% of all OTUs, while Proteobacteria and Acidobacteria were also the main bacteria in the soil, with amounts of 17.54\%-21.78\% and12.73\%-17.84\%, respectively (Fig. 2a). There was a difference in relative bacterial abundance before and after planting, both at the seedling and maturity stages, with a significant increase in Actinobacteria and a substantial decrease in Proteobacteria in the three cultivar groups compared with CK (Fig. 2a, Supplementary Fig. S2a). Some phyla, such as Actinobacteria, Acidobacteriota, and Gemmatimnadota, had significantly different relative abundances between cultivars. Actinobacteria differed among cultivars at the seedling stage but not significantly at maturity. Acidobacteriota of cultivar ZL differed from other cultivars at the seedling stage; Gemmatimnadota differed among cultivars at the maturity stage. In comparing different periods of the same cultivar, differences were found in Actinobacteria and Bacteroidota at both seedling and maturity stages (ANOVA. $P<$ 0.05) (Fig. 2a, Supplementary Fig. S2a). For fungi, compared with CK, the Ascomycota and Mortierellomycota in the cultivar groups showed a significant increase in both periods. In contrast, the Basidiomycota phylum showed a considerable decrease, most significantly in the N cultivar (ANOVA. $P<0.05$ ) (Fig. 2b, Supplementary Fig. S2b).

The soil community composition at the genus level was also different among cultivars. In the top 50 genera, the bacterial community was dominated by Arthrobacter, Rubrobacter, and Blastococcus. Sphingominas, Microlunatus, and Rubrobacterhad lower abundances in the CK group, while RB41, Paenibacillus, Streptomyces, and Pseudonocardia had lower abundances after planting. Comparison between seedling and maturity stages also revealed that Gaiella, Nocardioides, Pseudoncardia, and Blastococcus were generally less abundant at the seedling stage (Fig. 3a). There were 20 dominant species at the seedling stage and 22 dominant species at the maturity stage (average abundance $>1 \%$ ). By comparing the dominant species of the two varieties during the same period, further analysis showed that the bacterial community composition of cultivars N and QZ was similar but different from that of cultivar $Z L$ at the seedling stage. At the seedling stage, the abundance of Pseudonocardia and Blastococcus was significantly higher $(\mathrm{P}<0.05)$ in N and QZ compared with that in ZL (Fig. 3a,c); Streptomyces and Nocardioides in cultivar $N$ were significantly higher $(P<0.05)$ compared with those in ZL 9 (Fig. 3a,c). At maturity, Blastococcus and Microvirga were significantly higher $(\mathrm{P}<0.05)$ in cultivar $\mathrm{N}$ than in cultivar $\mathrm{QZ}$, while the relative abundance of Rubrobacter and $R B 41$ was relatively higher $(\mathrm{P}<0.05)$ in cultivar ZL (Fig. 3a,d). 
The fungal community composition at the genus level varied among cultivars at different periods. By analyzing the relative abundance of fungi at the genus level, the community was dominated by Tausonia, Gibberella, Pseudombrophila, Schizothecium and Mortierella (Fig. 3b). There were 12 dominant genera at the seedling stage and 14 dominant genera at the maturity stage (average abundance $>1 \%$ ). Comparisons indicated that Gibellulopsis, Monondicty, Sporoirmia, and Tausonia decreased after planting, especially in cultivar N (Fig. 3b), while the abundance of Pseudombrophila increased significantly $(p<0.05)$ (Fig. 3b,f). Tausonia had a higher relative abundance in cultivar ZL, while Monocillium and Coprinopsis had a higher relative abundance in cultivar QZ (Fig. $3 b)$. However, further analysis showed that the bacterial community composition of dominant genera in cultivars $\mathrm{N}$ and QZ was similar at the seedling stage (Fig. 3e). At maturity, the abundance of Rhizoctonia was significantly higher $(p<0.05)$ in cultivars (Fig. 3b); the relative abundances of Gibberalle and Chaetomium differed significantly $(p<0.05)$ between cultivars ZL and QZ (Fig. 3f). Compared with bacteria, the fungal differences were not particularly significant.

\section{Topological properties of the bacterial and fungal co-occurrence network}

Co-occurrence networks were built to construct high-throughput sequencing data at the genus level. Network plots revealed that the three cultivars had different microbial co-occurrence network structures. The modular architecture was used as the coloring unit to visualize the ecological network of bacteria and fungi. Modules with less than $1 \%$ of nodes are shown in gray, with the node size proportional to the corresponding relative abundance. The same module represents the same trend of change. The red edges represent positive correlations, and the blue and green edges represent negative correlations.

The complexity of the co-occurrence network varied depending on both the variety and period. Different $\mathrm{R}^{2}$ values for all groups indicate that the networks formed possessed scale-free properties (Table 1). The network was looser at the seedling stage than at the maturity stage, suggesting that the network was more consistent at maturity (Fig. 4a). Fungal communities were compact at the seedling stage but loose at maturity (Fig. 4b). The network was dominated by red lines in the bacterial and fungal networks, indicating that positive interactions were higher than negative interactions. However, this was not the case for fungi in the mature stage of the $\mathrm{N}$ species (Fig. 4a,b).

Moreover, the average clustering coefficients, average path distance, and other parameters were also different between the twelve networks (Table 1). Comparing the three bacterial networks at the seedling stage, we discovered that the average path length (GD) of the ZL network was longer than those of the $\mathrm{N}$ and QZ networks, while the average connectivity and clustering coefficients (avgCC) of the N network were relatively higher. QZ had a higher average degree (avgK) and a shorter average path distance (GD) (Table 1). However, in regard to maturity, the opposite was true (Fig. 4a, Table 1). For fungi, the network of the QZ cultivar was the tightest in the two periods (Fig. $4 a$, Table 1). Overall, network analyses indicated higher stability properties in the bacterial networks than in the fungal networks (Fig. 4a, Table 1).

Centrality is a concept commonly used in network analysis to express how each point in a network is related to other issues in the network. The nodes of maximum stress centrality were also different in all groups, such as Aridibacter, Phyllobacterium, and Pedomicrobium; none of these genera were found in the top 50 genera (Table 1). The degree of a node indicates the number of nodes in the network that are directly connected to that node, with higher connectivity indicating higher importance of the node in the overall network. The nodes with a maximum degree also varied in different cultivars, but the virtual nodes in the same cultivar in different periods always had consistency (Table 1). 
Different nodes may play distinctly different topological roles in a network, which is shown in Supplementary Table S4 in our experiment. Different cultivars had different key nodes at different periods. At the seedling stage, the connector found in cultivar N was Herbinix, and the module hubs were Bacillus, Gaiella, RB41, and so on. In cultivar QZ, Bacillus and Phyllobacteria were the module hubs. Halocella and Psychrobacillua (a kind of bacillus) were the module hubs in cultivar ZL (Supplementary Table S4). At maturity, the connector found in cultivar N was Rhpdpcytophage, the module hubs were Asanoa, Conexobacter, and Sporosarina. In cultivar QZ, Microbacterium and Nannocystis were the module hubs. Galella, Herpetosiphon, and Bdellourbrio were the module hubs and connectors in cultivar ZL (Supplementary Table S5). Similar results were found for the Z-P table of fungi, with different cultivars having different key nodes (Supplementary Tables S6, S7).

Table 1. Topological properties of co-occurring bacterial and fungal networks of two periods obtained among the rhizosphere.

\begin{tabular}{|c|c|c|c|c|c|c|c|c|c|}
\hline & Community & $\begin{array}{l}\text { Total } \\
\text { nodes }\end{array}$ & $\begin{array}{l}\text { Total } \\
\text { links }\end{array}$ & $\begin{array}{l}\mathrm{R}^{2} \\
\text { square } \\
\text { of } \\
\text { power- } \\
\text { law }\end{array}$ & $\begin{array}{l}\text { Average } \\
\text { degree } \\
\text { (avgK) }\end{array}$ & $\begin{array}{l}\text { Average } \\
\text { clustering } \\
\text { coefficient } \\
\text { (avgCC) }\end{array}$ & $\begin{array}{l}\text { Average } \\
\text { path } \\
\text { distance } \\
\text { (GD) }\end{array}$ & $\begin{array}{l}\text { Nodes } \\
\text { with } \\
\text { max } \\
\text { degree }\end{array}$ & $\begin{array}{l}\text { Nodes } \\
\text { with max } \\
\text { stress } \\
\text { centrality }\end{array}$ \\
\hline \multirow[t]{6}{*}{ Bacteria } & $\mathrm{N}$ & 471 & 867 & 0.914 & 3.682 & 0.222 & 8.062 & OTU838 & OTU392 \\
\hline & QZ & 465 & 857 & 0.894 & 3.686 & 0.21 & 6.037 & OTU509 & OTU402 \\
\hline & ZL & 551 & 808 & 0.878 & 2.933 & 0.206 & 10.222 & OTU604 & OTU900 \\
\hline & N2 & 469 & 762 & 0.926 & 3.249 & 0.17 & 8.361 & OTU838 & OTU58 \\
\hline & QZ2 & 498 & 716 & 0.833 & 2.876 & 0.18 & 8.926 & OTU319 & OTU984 \\
\hline & ZL2 & 445 & 1010 & 0.865 & 4.539 & 0.176 & 7.925 & OTU604 & OTU248 \\
\hline \multirow[t]{6}{*}{ Fungi } & $\mathrm{N}$ & 152 & 448 & 0.835 & 5.895 & 0.289 & 3.517 & OTU195 & OTU56 \\
\hline & QZ & 139 & 375 & 0.781 & 5.396 & 0.253 & 3.377 & OTU356 & OTU356 \\
\hline & ZL & 180 & 284 & 0.802 & 3.156 & 0.172 & 6.683 & OTU104 & OTU104 \\
\hline & N2 & 210 & 314 & 0.709 & 2.99 & 0.186 & 6.691 & OTU150 & OTU150 \\
\hline & QZ2 & 155 & 288 & 0.831 & 3.716 & 0.19 & 4.525 & OTU6 & OTU6 \\
\hline & ZL2 & 177 & 226 & 0.869 & 2.554 & 0.131 & 7.36 & OTU202 & OTU324 \\
\hline
\end{tabular}

We constructed Spearman's analysis of the top 50 genera of well-functioning bacteria and pathogen-associated fungi to find the relationships between different bacteria and fungi (Fig. 4c). We discovered that Blastococcus RB4 1 was significantly positively $(P \leq 0.05)$ correlated with Bacillus and negatively correlated with Rhizoctonia; Gaiella was significantly negatively correlated $(P \leq 0.05)$ with Elin6055; and Microvirga was significantly negatively $(P \leq$ 0.05) correlated with Gribberella (Fig. 4c). Bacillus was the key node in both cultivars. In contrast, nodes in fungi with the maximum degree and stress centrality converged in the same cultivar; therefore, we compared the two and found more associations between key fungi than with Bacillus (Supplementary Fig. S3). Fungi and bacteria were in a state of mutual constraint and interdependence, and interestingly, pathogenic bacteria in fungi did not all show a negative correlation (Supplementary Fig. S4). 
FAPROTAX functional predictions were made for bacteria in the soil at different periods, with the highest abundance of bacteria related to chemoisotropy and aerobic-chemoheterotrophy (Fig. 5a, Supplementary Fig. S5), indicating that the microorganisms were closely related to plant metabolism. Microorganisms related to the degradation of aromatic compounds and cellulose decomposition were more abundant in $\mathrm{N}$, microorganisms related to nitrogen fixation, and microorganisms related to lignin degradation were more abundant in QZ (Fig. 5a). In the seedling stage, ZL was closely related to nitrate reduction and nitrate respiration, and microorganisms related to photoautotrophy were less abundant at maturity than at the seedling stage, a phenomenon in contrast to cultivar N (Fig. 5a, Supplementary Fig. S5). FUNGuild function predictions for fungi at both periods revealed that the QZ and N cultivars had fewer fungi associated with plant pathogens at maturity with QZ. Cultivars had the fewest fungi associated with plant pathogens at maturity (Fig. 5b,c).

\section{Discussion}

The interaction between plants and microorganism communities is a critical driving issue for ecosystem functions(Graham et al. 2014; Wu et al. 2019). Soil microorganisms play a significant role in the decomposition of organic matter (Collado et al. 2019), nutrient acquisition (Chibucos 2009), and soil nutrient dynamics (Hou et al. 2018). Many studies have shown a direct relationship between crop genotype and interroot microbes. Consistent with previous studies, our study also ascertained these patterns. We conjectured that differences in microbial community structure in maize at different times might be related to genotype and designed experiments to test this hypothesis. We conducted 16S rRNA gene and ITS gene amplicon sequencing to detect the diversity and structure of the maize rhizosphere microbiota.

By analyzing the $a$ diversity and $\beta$ diversity of the microbial community, the results demonstrated that the microbial composition of maize soils differed among the three genotypes and was confirmed in both periods. $\mathrm{N}$ had the highest alpha diversity index (Fig. 1a,d), which may have a better degree of stability in the ecosystem(Xun et al. 2021). This suggested that the host genotype contributed to a considerable portion of the variation in maize rhizosphere microbial $a$ and $\beta$ diversity (Fig. 1b-c,e-f) (Peiffer et al. 2013).

To further explore the main manifestations of these differences, we analyzed the horizontal composition of the microbiota in the interroot microbial community structure of different maize genotypes. In all soil samples (Fig. 2a), Proteobacteria and Actinobacteria were the dominant phyla in soils, consistent with previous generation-sequencing studies on maize cultivated soils (Kong et al. 2020).

While many taxa were shared between different genotypes and soils, we also observed microbial taxa in which the soil community composition at the genus level was also different among cultivars (Fig. 3). For example, in the seedling stage, Blastococcus and Pseudonocardia were significantly higher $(P \leq 0.05)$ in N and QZ (Fig. 3c), and Pseudonocardia was reported to be mainly related to synthetic antibiotics (Caldera et al. 2019). In the maturity stage, Blastococcus, Pseudonocardia, and Microvirga were significantly higher $(P \leq 0.05)$ in N than in QZ, while RB41 was significantly higher $(P \leq 0.05)$ in ZL (Fig. 3d). A previous study showed that the abundance of Blastococcus and $R B 41$ in soil changed with salt stress. We suggested that the abundance of genera in ZL was more variable and that the cause of the variation in the abundance of ZL may be related to salt stress (Kloepper et al. 1980; Wang et al. 2019). 
Interestingly, we found that Blastococcus was positively correlated with Pseudonocardia, RB41, and Bacillus. Microviga was positively correlated with Springomonas(Zhou et al. 2016) but not with some genera (Pseudonocardia, RB41, and Bacillus) (Fig. 4c), probably because the association between the genera was in different patterns. We therefore hypothesized that different genotypes would lead to changes in the abundance of genera in the microbial community and might be more inclined to recruit microbes with the same trend required by the plant host. The fungal community was not as significant as the bacterial community in different cultivars (Fig. $3 e, f)$. We believe that genotype had a greater effect on the microbial community bacteria than fungi.

To further confirm our hypothesis, we conducted a co-occurrence network (Fig. 4a). Network analysis was performed to realize an integrated understanding of the bacterial community assembly rules reflecting ecological processes within the rhizosphere, such as cooperation, competition, and niche partitioning (Layeghifard et al. 2017). Moreover, correlation-based networks offered new insights into the characteristics of advanced bacterial network structures and keystone populations (Barberán et al. 2012). Bacterial and fungal networks differed across genotypes, which was confirmed in both periods. For example, QZ responded more quickly to changes in external environmental conditions and was more susceptible to changes in the external environment and less resistant to interference than ZL, which had an excellent buffering capacity for changes in environmental conditions (Fig. 4a,c) (Zhan et al. 2021). This suggested that different genotypes build their own unique microbial communities. The nodes with a maximum degree also varied in different cultivars, but in the same cultivar of different periods, they always had consistency (Table 1). This reinforced the idea that genotype mediates microbial changes.

We found different genotypes of connectors and module hubs and differences between periods. In the seedling stage, the connectors found in $\mathrm{N}$ were all related to functions such as cellulose degradation and plant growth and development (Koeck et al. 2016; Zhang et al. 2020). The connector found in QZ was related to bacterial exopolysaccharides (EPSs), which can provide the host plant with antioxidants and protection from corrosive pathogens (Supplementary Table S4) (Bouchotroch et al. 2000; Chi et al. 2019). The connector found in ZL was closely related to salt stress (Heng et al. 2019). Bacillus is a module hub in two cultivars. At maturity, the connectors found in cultivars were very different from seedlings (Supplementary Table S5). Therefore, we speculated that different genotypes of maize might build their unique microbial communities based on the unique characteristics of this variety. Notably, Blastococcus was less abundant in ZL than in the other two species but was an important node (Fig. 3c, Supplementary Table S5). None of the nodes of maximum stress centrality were found in the top 50 genera (Table 1). Some nodes with essential roles in the network are not dominant genera, revealing the vital role of rare species in the network (Deng et al. 2016; Feng et al. 2017). However, for fungi, many nodes with the maximum degree overlap with stress centrality; therefore, it is speculated that the fungal and bacterial communities may be in two different modes (Fig. 4c). We also found that fungi and bacteria were in a state of mutual constraint and interdependence, and interestingly, pathogenic in fungi did not all show a negative correlation with bacteria(Fig. 4c).

A variety of experimental studies have demonstrated the importance of biodiversity for system functioning (DelgadoBaquerizo et al. 2016; Hector et al. 2007; Wagg et al. 2014). The highest abundance of bacteria was related to chemoisotropy and aerobic chemoheterotrophy (Fig. 5a, Supplementary Fig. S5), indicating that microorganisms are closely related to plant metabolism. The variations in root exudates explained how these corn cultivars managed their rhizosphere bacterial ecosystems together with the core and cultivar-specific microbiota (Fig. 5a,

Supplementary Fig. S5)(Mendes et al. 2014). Ecological function predictions support network analysis, confirming a preference trend for plant-mediated microbial community change. Plant hosts of maize genotypes all prefer their corresponding ecological functions and recruit their specific interrooted microbes according to their preferences (Fig. 5a, Supplementary Fig. S5). Bacteria associated with plant pathogens were more abundant at the seedling 
stage but less abundant at maturity (Fig. 5b-c). Several recent studies have provided proof of this strategy in which surface pathogen infection can induce the assemblage of a plant-beneficial bacterial consortium in the root microbiome (Berendsen et al. 2018; Yuan et al. 2018). We therefore believe that young seedlings are more susceptible to pathogens than mature plants. Plants secrete interroot secretions that are involved in a 'call for help' strategy and actively engage their microbes to maximize their survival and growth when affected by external stresses, leading to an enrichment of beneficial bacteria that become essential members of the dominant network (Liu et al. 2019), and these also closely related to the characteristics of the plant.

\section{Conclusions}

In summary, our evidence suggested that different genotypes of maize regulated the rhizosphere microbial community structure in both periods, with secreting root exudates to build their unique microbial communities, depending on species unique characteristics. Genotype had a greater effect on the microbial community bacteria than fungi. The fungal and bacterial communities might be in two different modes and a state of interconnectedness. Our study further confirms the pattern of genotype-induced microbial community assembly. These findings offer a framework for the more development of methods aiming at engineering useful plant microbiomes via changes. By better understanding the causal relationship between genotypes and microbial communities and the relationship with species unique characteristics, we will probably be able to better inform methods for prospective designs, manipulate the structure, functioning of plant-associated microbiomes and provide a guiding direction for the subsequent construction of rhizosphere microbial communities relevant to maize growth and development.

\section{Declarations}

\section{Supplementary Materials:}

Supplementary materials include one Supplementary Fig. file and one Supplementary Table file. Supplementary Fig. file: Fig. S1: Rank abundance curve of the bacterial and fungal community. Fig. S2: Significance test of differences between three different rhizosphere maize groups based on Student's t test at the phylum level. Fig. S3: Spearman's analysis between nodes with maximum degree and stress centrality in fungi and Bacillus. Fig. S4: Spearman analysis of well-functioning bacteria and pathogen-associated fungi of the first fifty genera. Fig. S5: Kruskal-Wallis $\mathrm{H}$ test on the FAPROTAX functional (relative abundance $>0.5 \%$ ). Table S1: Soil physical and chemical properties at the study site.Table S2: Biological characteristics of different maize varieties. Table S3: The PCR amplification of 16S rRNA gene. Table S4: Connectors and module hubs of the bacterial seedling stage. Table S5: Connectors and module hubs of the bacterial maturity stage. Table S6: Connectors and module hubs of the fungal seedling stage. Table S7: Connectors and module hubs of the fungal maturity stage.

\section{Author Contributions:}

Conceptualization, Y.L. and Z.W.; methodology, Y.L. and Z.W.; software, Y.L. and Z.Q.; validation, Z.Q., W.X., Y.H. W.C. and Z.W.; resources, Z.W., Y.H. and W.C.; data curation, Y.L.; writing-original draft preparation, Y.L.; writing-review and editing, Y.L. and Z.W.; visualization, Y.L. and Z.Q.; supervision, Z.W. and W.X.; project administration, Z.W. and W.X.; funding acquisition, Z.W. All authors contributed to the article and approved the submitted version. All authors have read and agreed to the published version of the manuscript. 
Funding: This research was funded by the Key Research and Development Projects in Heilongjiang, China (GA21B007 and GZ20210014) and the Basic Research Fees of Universities in Heilongjiang Province, China (No. 135409103).

\section{Ethical approval: Not applicable.}

Consent to participate: Not applicable.

Consent for publication: Not applicable.

\section{Data Availability Statement:}

The datasets of the paper is deposited in NCBI under accession number PRJNA775859.

\section{Acknowledgments:}

We would like to thank the Qiqihar University, Heilongjiang Province, China for support of experimental site and helping in this study. And the data were analyzed on the free online platform of Majorbio Cloud Platform (www.majorbio.com).

\section{Conflflicts of Interest:}

The authors declare no conflflict of interest. The funders had no role in the design of the study; in the collection, analyses, or interpretation of data; in the writing of the manuscript, or in the decision to publish the results.

\section{References}

1. Agler M T et al (2016) Microbial Hub Taxa Link Host and Abiotic Factors to Plant Microbiome Variation. PLoS Biol 14: e1002352. https://doi.org/10.1371/journal.pbio.1002352

2. Barillot C D C, Sarde C O, Bert V, Tarnaud E, Cochet N (2013) A standardized method for the sampling of rhizosphere and rhizoplan soil bacteria associated to a herbaceous root system. Ann Microbiol 63: 471-476. https://doi.org/10.1007/s13213-012-0491-y

3. Bakker P, Doornbos R F, Zamioudis C, Berendsen R L, Pieterse C (2013) Induced Systemic Resistance and the Rhizosphere Microbiome. The Plant Pathology Journal 29:136-143. https://doi.org/10.5423/PPJ.SI.07.2012.0111

4. Barberán A, Bates S T, Casamayor E O, Fierer N (2012) Using network analysis to explore co-occurrence patterns in soil microbial communities. Isme j 6: 343-351. https://doi.org/10.1038/ismej.2011.119

5. Berendsen R L et al (2018) Disease-induced assemblage of a plant-beneficial bacterial consortium. Isme j 12 : 1496-1507. https://doi.org/10.1038/s41396-018-0093-1

6. Berg M, Koskella B (2018) Nutrient- and Dose-Dependent Microbiome-Mediated Protection against a Plant Pathogen. Current biology: CB 28: 2487. https://doi.org/10.1016/j.cub.2018.05.085

7. Bouchotroch S, Quesada E, Izquierdo I, Rodríguez M, Béjar V (2000) Bacterial exopolysaccharides produced by newly discovered bacteria belonging to the genus Halomonas, isolated from hypersaline habitats in Morocco. $J$ Ind Microbiol Biotechnol 24: 374-378. https://doi.org/10.1038/sj.jim.7000002

8. Brughmans T (2013) Thinking through networks: a review of formal network methods in archaeology. Journal of Archaeological Method \& Theory 20: 623-662. https://doi.org/10.1007/s10816-012-9133-8 
9. Bulgarelli D et al (2015) Structure and Function of the Bacterial Root Microbiota in Wild and Domesticated Barley. Cell Host Microbe 17: 392-403. https://doi.org/10.1016/j.chom.2015.01.011

10. Caldera E J, Chevrette M G, Mcdonald B R, Currie C R (2019) Local Adaptation of Bacterial Symbionts within a Geographic Mosaic of Antibiotic Coevolution. Appl Environ Microbiol 85: e01580-19. https://doi.org/10.1128/AEM.01580-19

11. Chandel R (2018) Response of maize (Zea mays L.): Crop to different planters. Poljoprivredna Tehnika 2: 61-72.

12. Chen S, Zhou Y, Chen Y, Gu J (2018) fastp: an ultra-fast all-in-one FASTQ preprocessor. Bioinformatics 34: i884i890. https://doi.org/10.1093/bioinformatics/bty560

13. Chi $Y$ et al (2019) Structure and molecular morphology of a novel moisturizing exopolysaccharide produced by Phyllobacterium sp. 921F. Int J Biol Macromol 135, 998-1005. https://doi.org/ 10.1016/j.ijbiomac.2019.06.019

14. Chibucos M C, Tyler B M (2009) Common themes in nutrient acquisition by plant symbiotic microbes, described by the Gene Ontology. BMC Microbiol 9: S6. https://doi.org/ 10.1186/1471-2180-9-S1-S6

15. Cline L C, Zak D R (2016) Soil microbial communities are shaped by plant-driven changes in resource availability during secondary succession. Ecology https://doi.org/96: 3374-3385. 10.1890/15-0184.1

16. Collado S, Oulego P, Suarez-Iglesias O, Diaz M (2019) Leachates and natural organic matter. A review of their biotreatment using fungi. Waste Manage 96: 108-120. https://doi.org/ 10.1016/j.wasman.2019.07.018

17. Delgado-Baquerizo $\mathrm{M}$ et al (2016) Microbial diversity drives multifunctionality in terrestrial ecosystems. Nat Commun 7: 10541. https://doi.org/10.1038/ncomms10541

18. Delmont $\mathrm{T} O$ et al (2011) Accessing the Soil Metagenome for Studies of Microbial Diversity. Appl Environ Microbiol 77: 1315-1324. https://doi.org/10.1128/AEM.01526-10

19. Deng $Y$ et al (2012) Molecular ecological network analyses. BMC Bioinformatics 13:113. https://doi.org/10.1186/1471-2105-13-113

20. Deng $Y$ et al (2016) Network succession reveals the importance of competition in response to emulsified vegetable oil amendment for uranium bioremediation. Environ Microbiol 18: 205-218.

https://doi.org/10.1111/1462-2920.12981

21. Edgar R C (2013) UPARSE: highly accurate OTU sequences from microbial amplicon reads. Nat Methods 10 : 996-998. https://doi.org/10.1038/NMETH.2604

22. Etesami H, Hosseini H M, Alikhani H A (2014) Bacterial biosynthesis of 1-aminocyclopropane-1-caboxylate (ACC) deaminase, a useful trait to elongation and endophytic colonization of the roots of rice under constant flooded conditions. Physiol Mol Biol Plants 20: 425-434. https://doi.org/10.1007/s12298-014-0251-5

23. Feng $\mathrm{K}$ et al (2017) Biodiversity and species competition regulate the resilience of microbial biofilm community. Mol Ecol 26: 6170-6182. https://doi.org/10.1111/mec.14356

24. Ferrando L, Scavino A F (2015) Strong shift in the diazotrophic endophytic bacterial community inhabiting rice (Oryza sativa) plants after flooding. FEMS Microbiol. Ecol 91: fiv104. https://doi.org/10.1093/femsec/fiv104

25. Gardener B, Weller D M (2001) Changes in Populations of Rhizosphere Bacteria Associated with Take-All Disease of Wheat. Appl Environ Microbiol. 67: 4414-4425. https://doi.org/10.1128/AEM.67.10.4414-4425.2001

26. Graham E B et al (2014) Do we need to understand microbial communities to predict ecosystem function? A comparison of statistical models of nitrogen cycling processes. Soil Biol Biochem 68: 279-282. https://doi.org/10.1016/j.soilbio.2013.08.023

27. Haney C H, Samuel B S, Bush J, Ausubel F M (2015) Associations with rhizosphere bacteria can confer an adaptive advantage to plants. Nat Plants 1: 15051. https://doi.org/10.1038/NPLANTS.2015.51

Page $12 / 17$ 
28. Hector A, Bagchi R (2007) Biodiversity and ecosystem multifunctionality. Nature 448: 188-190. https://doi.org/10.1038/nature05947

29. Heng S, Sutheeworapong A S, Prommeenate B P, Cheevadhanarak C S, Kosugi B A (2019) Complete Genome Sequence of Halocella sp. Strain SP3-1, an Extremely Halophilic, Glycoside Hydrolase- and BacteriocinProducing Bacterium Isolated from a Salt Evaporation Pond. Microbiology Resource Announcements 8: e0169601618. https://doi.org/10.1128/MRA.01696-18

30. Hou D et al (2018) Cultivar-specific response of bacterial community to cadmium contamination in the rhizosphere of rice ( Oryza sativa L.). Environ Pollut 241: 63-73. https://doi.org/ 10.1016/j.envpol.2018.04.121

31. Jiang $Y$ et al (2017) Plant cultivars imprint the rhizosphere bacterial community composition and association networks. Soil Biol Biochem 109:145-155. https://doi.org/ 10.1016/j.soilbio.2017.02.010

32. Junker B H, Schreiber F (2010) Analysis of biological networks. J Anat 215: 473. https://doi.org/ 10.1111/j.1469-7580.2009.01132.x

33. Kloepper J W, Leong J, Teintze M, Schroth M N (1980) Pseudomonas siderophores: A mechanism explaining disease-suppressive soils. Curr Microbiol 4: 317-320. https://doi.org/ 10.1007/BF02602840

34. Koeck D E et al (2016) Complete Genome Sequence of Herbinix luporum SD1D, a New Cellulose-Degrading Bacterium Isolated from a Thermophilic Biogas Reactor. Genome Announc 4: e00687-16. https://doi.org/10.1128/genomeA.00687-16

35. Kong $X$ et al (2020) Maize (Zea mays L. Sp.) varieties significantly influence bacterial and fungal community in bulk soil, rhizosphere soil, and phyllosphere. FEMS Microbiol Ecol 96:3. https://doi.org/10.1093/femsec/fiaa020

36. Layeghifard M, Hwang D M, Guttman D S (2017) Disentangling Interactions in the Microbiome: A Network Perspective. Trends Microbiol 25: 217-228. https://doi.org/10.1016/j.tim.2016.11.008

37. Liu H, Macdonald C A, Cook J, Anderson I C, Singh B K (2019) An Ecological Loop: Host Microbiomes across Multitrophic Interactions. Trends Ecol Evol 34: 1118-1130. https://doi.org/ 10.1016/j.tree.2019.07.011

38. Lu T et al (2018) Rhizosphere microorganisms can influence the timing of plant flowering. Microbiome 6:231. https://doi.org/10.1186/s40168-018-0615-0

39. Magoč T, Salzberg S L (2011) FLASH: fast length adjustment of short reads to improve genome assemblies. Bioinformatics 27: 2957-2963. https://doi.org/10.1093/bioinformatics/btr507

40. Mansotra P, Sharma P, Sharma S (2015) Bioaugmentation of Mesorhizobium cicer, Pseudomonas spp. and Piriformospora indica for Sustainable Chickpea Production. Physiol Mol Biol Plants 21: 385-393. https://doi.org/10.1007/s12298-015-0296-0

41. Mendes L W, Kuramae E E, Navarrete A A, van Veen J A, Tsai S M (2014) Taxonomical and functional microbial community selection in soybean rhizosphere. Isme j 8: 1577-1587. https://doi.org/10.1038/ismej.2014.17

42. Mendes R, Garbeva P, Raaijmakers J M (2013) The rhizosphere microbiome: significance of plant beneficial, plant pathogenic, and human pathogenic microorganisms. FEMS Microbiol Rev 37: 634-663. https://doi.org/10.1111/1574-6976.12028

43. Mendes R et al (2011) Deciphering the Rhizosphere Microbiome for Disease-Suppressive Bacteria. Sci 332:1097-1100. https://doi.org/10.1126/science.1203980

44. Müller D B, Vogel C, Bai Y, Vorholt Julia A (2016) The Plant Microbiota: Systems-Level Insights and Perspectives. Annu Rev Genet. 50: 211-234. https://doi.org/10.1146/annurev-genet-120215-034952

45. Nguyen N W et al (2016) FUNGuild: An open annotation tool for parsing fungal community datasets by ecological guild. Fungal Ecol 20:241-248. https://doi.org/10.1016/j.funeco.2015.06.006 
46. Peiffer J A et al (2013) Diversity and heritability of the maize rhizosphere microbiome under field conditions. Proc Natl Acad Sci U S A 110: 6548-6553. https://doi.org/10.1073/pnas.1302837110

47. Pozo M J, Azcón-Aguilar C (2007) Unravelling mycorrhiza-induced resistance.10:393-398. https://doi.org/10.1016/j.pbi.2007.05.004

48. Raaijmakers J M, Mazzola M (2016) Soil immune responses. Sci 352: 1392-1393. https://doi.org/10.1126/science.aaf3252

49. Reinhold-Hurek B, Bünger W, Burbano C S, Sabale M, Hurek T (2015) Roots shaping their microbiome: global hotspots for microbial activity. Annu Rev Phytopathol 53:403-424. https://doi.org/10.1146/annurev-phyto082712-102342

50. Ritpitakphong $U$ et al (2016) The microbiome of the leaf surface of Arabidopsis protects against a fungal pathogen. New Phytol 210: 1033-1043. https://doi.org/10.1111/nph.13808

51. Rolli E et al (2015) Improved plant resistance to drought is promoted by the root-associated microbiome as a water stress-dependent trait. Environ Microbiol 17: 316-331. https://doi.org/10.1111/1462-2920.12439

52. Saleem M, Law A D, Moe L A (2016) Nicotiana Roots Recruit Rare Rhizosphere Taxa as Major Root-Inhabiting Microbes. MicEc 71: 469-472. https://doi.org/10.1007/s00248-015-0672-x

53. Santos-Medellín C, Edwards J, Liechty Z, Nguyen B Sundaresan V (2017) Drought Stress Results in a Compartment-Specific Restructuring of the Rice Root-Associated Microbiomes mBio 8: e00764-00717. https://doi.org/10.1128/mBio.00764-17

54. Schlaeppi K, Dombrowski N, Oter R G, van Themaat E V L, Schulze-Lefert P (2014) Quantitative divergence of the bacterial root microbiota in Arabidopsis thaliana relatives. Proc Natl Acad Sci U S A 111: 585-592. https://doi.org/10.1073/pnas.1321597111

55. Simonin M et al (2020) Influence of plant genotype and soil on the wheat rhizosphere microbiome: evidences for a core microbiome across eight African and European soils. FEMS Microbiol. Ecol 96: fiaa067. https://doi.org/10.1093/femsec/fiaa067

56. Stackebrandt E, Goebel B M (1994) Taxonomic Note: A Place for DNA-DNA Reassociation and 16S rRNA Sequence Analysis in the Present Species Definition in Bacteriology. Int j syst bacteriol 44: 846-849.

57. Taguchi Y H, Oono Y (2005) Relational patterns of gene expression via nonmetric multidimensional scaling analysis. Bioinformatics 21: 730-740. https://doi.org/10.1093/bioinformatics/bti067

58. Teste F P et al (2017) Plant-soil feedback and the maintenance of diversity in Mediterranean-climate shrublands. Sci 355: 173. https://doi.org/10.1126/science.aai8291

59. Timm C M et al (2018) Abiotic Stresses Shift Belowground Populus -Associated Bacteria Toward a Core Stress Microbiome. mSystems 3: e00070. https://doi.org/10.1128/mSystems.00070-17

60. Vivanco L, Rascovan N, Austin A T (2018) Plant, fungal, bacterial, and nitrogen interactions in the litter layer of a native Patagonian forest. PeerJ 6: e4754. https://doi.org/10.7717/peerj.4754

61. Wagg C, Bender S F, Widmer F, van der Heijden M (2014) Soil biodiversity and soil community composition determine ecosystem multifunctionality. Proc Natl Acad Sci U S A 111: 5266-5270.

https://doi.org/10.1073/pnas.1320054111

62. Wang M, Chen S, Chen L, Wang D (2019) Responses of soil microbial communities and their network interactions to saline-alkaline stress in Cd-contaminated soils. Environ Pollut 252: 1609-1621.

https://doi.org/10.1016/j.envpol.2019.06.082

Page 14/17 
63. Wang Q, Garrity G M, Tiedje J M, Cole J R (2007) Naive Bayesian classifier for rapid assignment of rRNA sequences into the new bacterial taxonomy. Appl Environ Microbiol 73: 5261-5267.

https://doi.org/10.1128/AEM.00062-07

64. Wu R, Cheng X, Zhou W, Han H (2019) Microbial regulation of soil carbon properties under nitrogen addition and plant inputs removal. PeerJ 7: e7343. https://doi.org/10.7717/peerj.7343

65. Xu L et al (2015) Effects of Interactions of Auxin-Producing Bacteria and Bacterial-Feeding Nematodes on Regulation of Peanut Growths. PLoS One 10: e0124361. https://doi.org/ 10.1371/journal.pone.0124361

66. Xun W et al (2021) Specialized metabolic functions of keystone taxa sustain soil microbiome stability. Microbiome 9: 35. https://doi.org/10.1186/s40168-020-00985-9

67. Yu K, Pieterse C, Bakker P, Berendsen R L (2019) Beneficial microbes going underground of root immunity. Plant Cell and Environment 42: 2860-2870. https://doi.org/10.1111/pce.13632

68. Yuan $\mathrm{J}$ et al (2018) Root exudates drive the soil-borne legacy of aboveground pathogen infection. Microbiome 6: 156. https://doi.org/10.1186/s40168-018-0537-x

69. Zachow C, Muller H, Tilcher R, Berg G (2014) Differences between the rhizosphere microbiome of Beta vulgaris ssp. maritima-ancestor of all beet crops-and modern sugar beets. Front Microbiol 5:415. https://doi.org/10.3389/fmicb.2014.00415

70. Zgadzaj R et al (2016) Root nodule symbiosis in Lotus japonicus drives the establishment of distinctive rhizosphere, root, and nodule bacterial communities. Proc Natl Acad Sci U S A 113: E7996-E8005. https://doi.org/10.1073/pnas.1616564113

71. Zhalnina K et al (2018) Dynamic root exudate chemistry and microbial substrate preferences drive patterns in rhizosphere microbial community assembly. Nature microbiology 3:470-480 https://doi.org/ 10.1038/s41564018-0129-3

72. Zhan P et al (2021) Plant litter decomposition in wetlands is closely associated with phyllospheric fungi as revealed by microbial community dynamics and co-occurrence network. Sci Total Environ 753: 142194. https://doi.org/10.1016/j.scitotenv.2020.142194

73. Zhang Y, Hu A, Zhou J, Zhang W, Li P (2020) Comparison of bacterial communities in soil samples with and without tomato bacterial wilt caused by Ralstonia solanacearum species complex. BMC Microbiol 20: 89. https://doi.org/10.1186/s12866-020-01774-y

74. Zhou LS, Li H, Zhang Y, Han S, Xu H (2016) Sphingomonas from petroleum-contaminated soils in Shenfu, China and their PAHs degradation abilities. Braz J Microbiol 47: 271-278. https://doi.org/10.1016/j.bjm.2016.01.001

75. Zhou J et al (2020) Characterization of the core microbiome in tobacco leaves during aging. Microbiologyopen 9:e984. https://doi.org/10.1002/mbo3.984

\section{Figures}

\section{Figure 1}

Alpha diversity and beta diversity of bacterial and fungal communities for three cultivars in two growth periods. (a), Simpson diversity, Shannon diversity, and the Chao1 diversity of the bacterial level at the seedling and maturity stage; and (d), Simpson diversity, Shannon diversity, and the Chao1 diversity of the fungal level at the seedling and 
maturity stage. NMDS analysis of the bacterial and fungal community composition at the genus level based on Bray-Curtis distance. (b), NMDS analysis of bacterial level at the seedling stage; (c), NMDS analysis of bacterial level at the maturity stage; (e), NMDS analysis of fungal level at the seedling stage; and (f), NMDS analysis of fungal level at the maturity stage. Significances between different groups were compared using Wilcoxon's test, with the results indicated on the top ( $P \leq 0.05$ is marked as *, $P \leq 0.01$ is marked as **, and $P \leq 0.001$ is marked as ***). The abscissa is the group name, and the ordinate is the average value of the index.

\section{Figure 2}

Taxonomic assignments and percent of community abundance at the phylum level in the rhizosphere soil of different cultivars. (a) The average relative abundance of the bacterial community. (b) The average relative abundance of the fungal community.

\section{Figure 3}

Taxonomic assignments and percent of community abundance at the genus level in the rhizosphere soil of different cultivars. $(a, b)$ Heatmap of the soil bacterial groups and fungal groups of the top 50 genera. (c-f) Significance test of differences between three different rhizosphere maize groups with dominant species based on Student's $t$ test at the genus level ( $P \leq 0.05$ is marked as *, $P \leq 0.01$ is marked as **, and $P \leq 0.001$ is marked as ***).

\section{Figure 4}

Ecological networks of fungal and bacterial communities and topological properties. (a) Ecological networks of bacterial communities. (b) Ecological networks of fungal communities. Every node signifies a genus. Nodes area unit colored by modules. Blue and green lines indicate positive relationships between two individual nodes, and red lines indicate negative relationships. (c) Spearman analysis of well-functioning bacteria and pathogen-associated fungi at the level of the first fifty genera ( $P \leq 0.05$ is marked as $*, P \leq 0.01$ is marked as **, and $P \leq 0.001$ is marked as $* \star *)$.

\section{Figure 5}

FAPROTAX functional and FUNGuild functional predictions of different cultivars. (a) FAPROTAX function of three cultivars of two periods. (b) FUNGuild function predictions for fungi of two periods. (c) Analysis of the plant pathogen of three cultivars in fungi based on Student's t test $(P \leq 0.05$ is marked as *, $P \leq 0.01$ is marked as **, and $P \leq 0.001$ is marked as ***).

\section{Supplementary Files}

This is a list of supplementary files associated with this preprint. Click to download. 
- Graphicalabstract.png

- renamed6cb3a.docx

- renamedd6334.docx 